Population biology

\section{Mechanisms of coexistence}

\section{Wallace Arthur}

Is interspecific competition an important determinant of major community properties such as species diversity and, if so, what sort of influence does it have? Ecologists have long sought to answer this question, and have approached it in various ways. One aspect of the problem is determining how common competition is in nature in the first place. A diverse array of field experiments ${ }^{1.2}$ shows that it is indeed reasonably common, though certainly not universal, among closely related sympatric species. Another aspect of the problem is elucidating the population dynamics of competition, that is, attempting to answer the question of how competition 'works' in those systems in which it is known to be taking place. It is this aspect of the problem that is addressed by Karl Rothhaupt on page 660 of this issue ${ }^{3}$, in a fascinating series of experiments on two competing species of rotifer.

Of course, there have been many previous attempts to elucidate the population dynamics of competition, from the classic work of Gause ${ }^{4.5}$ onwards. But many earlier authors failed to investigate the biological mechanisms of competition and coexistence, instead examining only the compatibility of their experimental data with models of the Lotka-Volterra genre which are not, themselves, mechanistically explicit. Others - including Gause himself - postulated mechanisms of coexistence but did not quantify them or carry out the necessary experimental protocol $^{6}$ to distinguish the proposed mechanism from possible alternatives. Given this state of affairs, a particularly useful approach in current studies of competition is the comparison of experimentally derived data with models, such as those of Tilman ${ }^{7}$, which are mechanistically explicit in their approach. This is precisely what Rothhaupt sets out to do.

His experimental system involves two closely related species of herbivorous rotifer, Brachionus rubens and B. calyciflorus, which eat two species of alga - one species of Chlamydomonas and one of Monoraphidium. Both species of rotifer eat both algal species, but $B$. rubens prefers and does better on Monoraphidium, whereas the reverse is true of $B$. calyciflorus. In a four-component mixture containing both consumers and both resources, exploitative competition occurs between the rotifers, and the population dynamics of their competitive interaction can be followed for a sufficient time to determine whether the outcome is stable coexistence or competitive exclusion. Rothhaupt performs twelve such experiments, each under different resource conditions. In some cases, $B$. rubens competitively excludes its congener; in others the situation is reversed, with $B$. calyciflorus winning; and in others the two rotifers reach a state of stable coexistence.

Rothhaupt then assesses the compatibility of his experimental results with

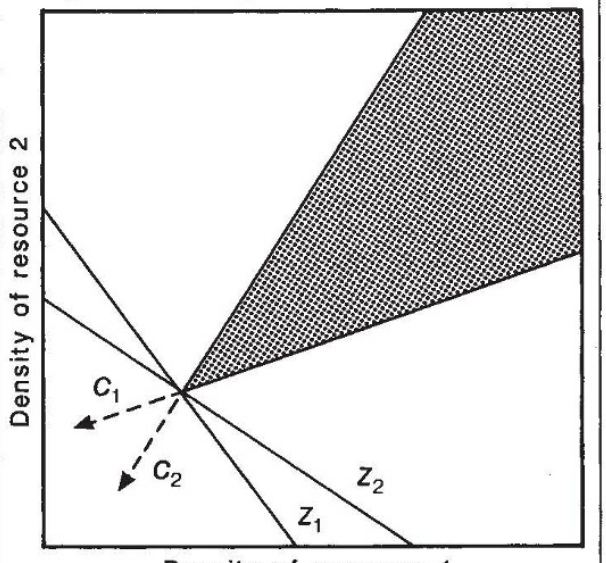

Density of resource 1

The division of the resource plane into a zone of predicted coexistence of the consumers (shaded) and a complementary zone in which other outcomes are predicted is achieved by projecting the consumption vectors $\left(c_{1}\right.$ and $\left.c_{2}\right)$ through the intersection of the zero net growth isoclines $\left(z_{1}\right.$ and $\left.z_{2}\right)$. In the shaded zone, the joint effect of resource supply and consumption is such as to move the resource densities towards the equilibrium point ${ }^{7}$.

Tilman's mechanistic theory ${ }^{7}$ of exploitative competition, where the conditions for stable coexistence are phrased in terms of resource supply and consumption, rather than in terms of abstract 'competition coefficients'. Specifically, Tilman's approach for a two-consumer, tworesource system involves subdivision of a resource plane - a two-dimensional space bounded by the densities of the resource species - into a zone in which coexistence is predicted and a complementary zone in which it is not. The way in which the resource plane is divided into these zones is complex ${ }^{7}$, and I do not have the space to explain it fully here, but the two relevant statistics (see figure) are the consumers' zero-net-growth isoclines (which join up points of zero population growth) and their consumption vectors (which indicate the effect on the resource densities of the consumers' feeding activities). Superimposing his results on to the appropriate resource plane, Rothhaupt finds a high degree of correspondence between prediction and observation. In five out of six cases where coexistence is predicted, it is found to occur, and in all six cases where competitive exclusion is predicted, one species does indeed exclude the other. Moreover, which species wins in the exclusion cases is also predicted by the theory, and in all cases the 'right' species wins.

Although this is an impressive correspondence between theory and experiment, some questions remain unanswered. Foremost among these is the question of how error-prone measurement of the consumption vectors is under any given set of conditions, and also how sensitive these vectors are to slight changes in extrinsic variables such as temperature. Because the consumption vectors delimit the zone of predicted coexistence, such questions are of crucial importance. The degree of error associated with measurements made under a single set of conditions is important in the interpretation of laboratory studies. It is particularly important in those, such as Rothhaupt's, where some of the resource treatments are perilously close to the edge of the zone of predicted coexistence. The degree of sensitivity of the consumption vectors to changes in extrinsic conditions is relevant to the application of this method to natural populations. If the zone of predicted coexistence alters its size and shape on a timescale less than that on which the competitive interaction normally delivers its end-result (exclusion or stable equilibrium), then considerable difficulties will be encountered. If not, then the method could be applicable to the field situation with no more than the usual additional difficulties associated with the laboratory-to-field transition.

Such difficulties aside, the combination of experimental studies (in both field and laboratory) with mechanistically explicit models provides a promising approach for the continuing elucidation of how interspecific competition 'works'. How useful this elucidation will be in the study of the main features of community structure depends on, among other things, the extent to which the population dynamics of competition in radically different groups of consumers follow the same rules. Many careful experimental studies are necessary before we can discover whether general rules for competition are a reality of nature or a theoretician's dream.

1. Schoener, T.W. Am. Nat. 122, 240-285 (1983).

2. Connell, J.H. Am. Nat. 122, 661-696 (1983)

3. Rothhaupt, K.O. Nature 333, 660-662 (1988)

4. Gause, G.F. The Struggle for Existence (Williams and Wilkins, Battimore, 1934).

5. Gause, G.F. Act. Sci. et Ind. 277 (1935)

6. Arthur, W. The Niche in Competition and Evolution (Wiley, Chichester, 1987).

7. Tilman, D. Resource Competition and Community Structure (Princeton University Press, 1982).

Wallace Arthur is in the School of Biology, Sunderland Polytechnic, Sunderland SR1 3SD, $U K$. 